\title{
The Halo Mass Distribution of Field and Cluster Early-Type Galaxies
}

\author{
Marcel P. Bergmann \\ NOAO Gemini Science Center, P.O. Box 26732, Tucson, AZ 85726 \\ Amy Forestell \& Karl Gebhardt \\ Astronomy Department, University of Texas, Austin \\ Inger Jørgensen \\ Gemini Observatory, Hilo, Hawaii
}

\begin{abstract}
We describe an ongoing program to study the halo kinematics of a broad sample of early-type galaxies using integrated light measurements obtained with the Hobby-Eberly ${ }^{1}$ and Gemini $^{2}$ Telescopes.
\end{abstract}

\section{Motivation and Background}

We have begun a program to study the halo kinematics of a broad sample of early-type galaxies covering a large range of masses and local environment. Modern cosmological simulations have the resolution to probe the evolutionary histories of galaxies in great detail; differences are predicted in the halo kinematics of galaxies which form via major mergers, multiple minor mergers, or continued slow accretion (Meza et al. 2003). Our observations, via comparison with these simulations, will allow us to ascertain the dominant processes of mass assembly, and their relation to local galaxy environment.

Observational difficulties have limited previous work to the nearest and most massive galaxies, and this may bias our understanding of the galaxy evolution process. Our deep integrated light observations allow us to study a sample of galaxies which are further away, and of lower mass, and which may be more representative of the galaxy population as a whole.

\footnotetext{
${ }^{1}$ The Hobby-Eberly Telescope is operated by McDonald Observatory on behalf of the University of Texas at Austin, The Pennsylvania State University, Stanford University, LudwidMaximilians-Universitat Munchen, and Georg-August-Universitat Gottingen.

${ }^{2}$ Part of this project is based on observations obtained at the Gemini Observatory, which is operated by AURA, INC., under a cooperative agreement with the NSF on behalf of the Gemini Partnership: The NSF (USA), PPARC (UK), NRC (Canada), CONICYT (Chile), ARC (Australia), CNPq (Brazil) and CONICET (Argentina).
} 
Table 1. The Galaxy Sample (mean values taken from LEDA database)

\begin{tabular}{lcccll}
\hline Galaxy & $\begin{array}{c}\mathrm{M}_{\mathrm{B}} \\
(\mathrm{mag})\end{array}$ & $\begin{array}{c}\sigma_{0} \\
\left(\mathrm{~km} \mathrm{~s}^{-1}\right)\end{array}$ & $\begin{array}{c}\mathrm{cz}_{\mathrm{hel}} \\
\left(\mathrm{km} \mathrm{s}^{-1}\right)\end{array}$ & environment & $\begin{array}{l}\text { Telescope/ } \\
\text { Instrument }\end{array}$ \\
\hline NGC 1272 & -21.6 & 300 & 3830 & cluster & Gemini/GMOSN \\
NGC 2832 & -22.4 & 340 & 6880 & cluster & HET/LRS \\
NGC 4406 & -20.8 & 250 & -250 & cluster & HET/LRS \\
NGC 4889 & -22.4 & 400 & 6485 & cluster & HET/LRS \\
NGC 661 & -20.8 & 180 & 3850 & field & HET/LRS \\
NGC 1132 & -21.8 & 240 & 6950 & field & Gemini/GMOSN \\
NGC 3617 & -19.0 & 90 & 2200 & field & Gemini/GMOSS \\
NGC 6702 & -21.4 & 185 & 4750 & field & HET/LRS \\
NGC 7454 & -19.7 & 110 & 2000 & field & Gemini/GMOSN \\
\hline \hline
\end{tabular}

\section{Observations \& Data}

We have obtained deep longslit spectroscopy of the integrated stellar light in a sample of nine field and cluster early-type galaxies (Table 1). The data have been obtained with the Marcario Low Resolution Spectrograph on the HobbyEberly Telescope (HET), and with the two Gemini Multi-Object Spectrographs (GMOS, Hook et al. 2002) at Gemini North and Gemini South. The spectra are sufficiently deep to measure line-of-sight velocity dispersion profiles, and derive kinematic parameters $(\mathrm{v}, \sigma, \mathrm{h} 3, \mathrm{~h} 4)$ at $\mathrm{r}>2 \mathrm{R}_{\mathrm{e}}$ (local surface brightness fainter than $\mu_{\mathrm{B}} \sim 24.5$ ), on two axes: the major axis and position angle near the minor axis.

\section{Models and First Results: NGC 6702}

We use an orbit-based method that provides the most general axisymmetric model (Gebhardt et al. 2003). It fits the velocity profiles directly using a potential derived from the light profile, assuming a constant $\mathrm{M} / \mathrm{L}$, and a dark halo. We have used two profiles: a logarithmic potential and the NFW profile (Navarro, Frenk, \& White 1996). We have begun our modeling with the field galaxy NGC 6702 . The no dark halo model is strongly excluded by the data. The best fitting model has an orbital structure which is slightly radial throughout most of the galaxy (similar to what Cretton et al. (1999) find in their sample) and then becomes tangential at large radii.

\section{References}

Cretton, N., de Zeeuw, P.T., van der Marel, R.P., \& Rix, H.-W. 1999, ApJS, 124,383

Gebhardt, K. et al. 2003, ApJ, 583, 92

Hook, I. et al. 2002, Proceedings of the SPIE, 4841, 1645

Meza, A., Navarro, J., Steinmetz, M., \& Eke, V. 2003, ApJ, 590, 619

Navarro, J., Frenk, C.S., \& White, S.D.M. 1996, ApJ, 462, 563 (NFW) 